\title{
Some Implications of Mathematical Analyses of Epidemics
}

\section{Joel K Weltman*}

Department of Medicine, School of Medicine, Alpert Brown University, Providence, RI, 02912, USA

Keywords: Epidemics; Influenza; Dynamic systems; Chaos; Quasispecies; Logistic; Sigmoidal

\section{Introduction}

Mathematical analysis is a powerful tool that facilitates conceptualization and understanding of epidemics of infectious diseases [1]. The time-course of cumulative case count in epidemics is welldescribed by the sigmoidal shape of discrete and continuous logistic functions. For example [2], the Pearson coefficient ' $r$ ' for correlation between the observed cumulative sequence count and the computed values of logistic-type functions varied from a minimum of 0.9495 to a maximum of 0.9991 for pandemic influenza A (H1N1) pdm09 (pH1N1) hemagglutinin (HA) sequences collected at 23 geographic locations distributed world-wide $(\mathrm{p}<2.22 \mathrm{e}-16$ at each location). A normalized version of this logistic function is given below as equation 1 :

$$
Y=1 /\left(1+\mathrm{e}^{-\mathrm{at}}\right)
$$

Plots of values of $\mathrm{Y}$ and its first 5 derivatives with respect to time $(\mathrm{t})$ are shown in figure 1 with parameter (a) varying from 0 to 2.0. The results of variation of the non-linear parameter (a) in figure 1 reflect a framework for conceptualization and modeling a smooth flow of mutant subsets, probabilistically produced as quasispecies [3] and moving those quasispecie mutants through space and time in laminas of evolutionary viral trajectories. Because of their simplicity and goodness-of-fit to the observed data, logistic and other sigmoidal functions are useful components of differential and difference equations for the analysis of influenza and other infectious epidemics.
The fourth and fifth derivatives ( $Y^{\prime \prime \prime}$ and $Y^{\prime \prime \prime \prime ') ~ o f ~} Y$ shown in figure 1 are of the type of "jerk", "jounce" and "swing" functions associated with turbulence of fluid flow, instability of electrical circuits and irregularity of pendulum motion $[4,5]$. Furthermore, it has been reported that chaotic regions have been detected in SIS ODE epidemic models [6]. The biological significance of these mathematical disturbances of evolutionary trajectory remains to be determined.

\section{Acknowledgments}

Brown University Center for Computing and Visualization (CCV) for provided computer facilities and ancillary support for this research. Brown University Center for Computational Molecular Biology (CCMB) provided their support.

\section{References}

1. Luenberger DG (1979) Introduction to Dynamic Systems. New York: John Wiley \& Sons 26: 376-378.

2. Thompson WA, Weltman JK (2012) Bioinformatic Analyses of 2009-2010 Pandemic H1N1 Influenza A Hemagglutinin Subsets. J Med Microbiol Diagn.

3. Eigen M, McCaskill J, Peter Schuster (1988) Molecular Quasi-Species. J Phys Chem 92: 6881-6891.

4. Sprott JC (1997) Some simple chaotic jerk functions. Amer J Phys 65: 537-543.

5. Kopell N, Washburn R Jr (1982) Chaotic motions in the two-degree of freedom swing equations. IEEE Transactions on Circuits and Systems, Special Issue on Power Systems. 29: 738-746.

6. Das P, Mukandavire Z, Chiyaka C, Sen A, Mukherjee D (2009) Bifurcation and Chaos in a S-I-S Epidemic Model. Differential Equations and Dynamical Systems 17: 393-417.
*Corresponding author: Joel K Weltman, Department of Medicine, School of Medicine, Alpert Brown University, Providence, RI, 02912, USA E-mail: joel_weltman@brown.edu

Received October 15, 2012; Accepted October 16, 2012; Published October 18 2012

Citation: Weltman JK (2010) Some Implications of Mathematical Analyses of Epidemics. J Med Microb Diagn $\square:[e 115$. doi:10.4172/2161-0703.1000e115

Copyright: (c) 2013 Weltman JK. This is an open-access article distributed under the terms of the Creative Commons Attribution License, which permits unrestricted use, distribution, and reproduction in any medium, provided the original author and source are credited. 

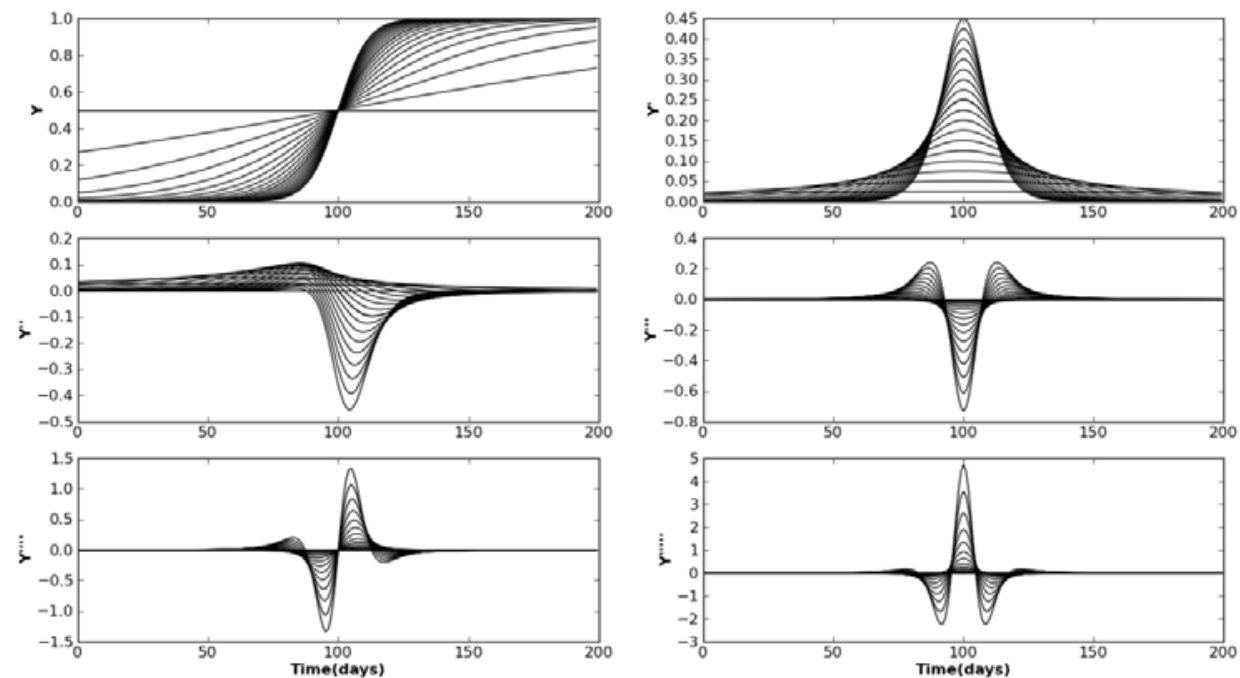

Figure 1: Time-Dependence of a Continuous Logistic-Function and its Derivatives.

Values of the logistic function $(Y)$ and the first five of its derivatives with respect to time (Y', Y", $Y^{\prime \prime \prime}, Y^{\prime \prime \prime \prime, ~} Y^{\prime \prime \prime \prime ') ~ a r e ~ o n ~ t h e ~ o r d i n a t e s . ~ T i m e ~ i s ~ d e n o t e d ~}$ on the abscissas. The function $(\mathrm{Y})$ is given in equation 1. Parameter (a) was varied from 0.0 to 2.0 in increments of 0.1 . Time derivatives of equation 1 were obtained with Maple 15.01 (Maplesoft, a division of Waterloo Maple, Inc.). Graphs were plotted with Python 2.7.3 \{EPD 7.3-1 (64-bit)\}, SciPy and matplotlib. 